\title{
Uncertainty and Practical Reasoning in Clinical Psychiatry and Neuropsychiatry
}

\author{
Andrew A. Nierenberg, MD
}

Psychiatrists and neurologists, like all physicians, make clinical decisions under conditions of uncertainty. ${ }^{1,2}$ Many questions arise during clinical care. What should clinicians do for patients whose diagnosis does not quite meet formal diagnostic criteria? What treatment works best for which patient? Which competing treatment should one choose? Should multiple treatments be started simultaneously or should treatments be given in sequence? How should comorbid conditions be addressed? When should medication be given to someone in distress when the distress is caused by severe psychosocial stress (such as bereavement)? When should a clinician declare that a treatment is failing? Should the trial be extended with the hope that some patients will eventually respond? What would be the optimal course of action after the first, second, third, or subsequent treatment fails? How does one best weigh benefits and risks, and integrate the clinician's perception of benefits and risks with the patient's perceptions? In addition to strategies (what to do), these questions also bring up tactical issues (how to do it) which are usually not covered in the literature. ${ }^{3}$

When clinicians face tactical micro-decisions, they are frequently uncertain about the best course of action. With sufficient information from the literature, we can estimate the probability (and, perhaps, beyond point probability, the 95\% confidence interval) of an outcome for the group that a patient belongs to, but not the probability of that patient's individual outcome. ${ }^{4}$ Clinicians apply group data (nomothetic) from the literature to the particular data (ideographic) from individual patients and hope for the best outcome. ${ }^{5}$ Few, if any, studies bring certainty to medical decision-making for any individual patient. Most clinical trials are designed to explain ("does the intervention change the target outcome?") rather than guide treatment ("does the intervention work when used in normal practice?"). ${ }^{6}$ The problem is that results from explanatory trials are used for treatment decisions, which is not the purpose of explanatory trials.

To decrease uncertainty, clinicians turn to multiple sources to make optimal clinical decisions to increase the probability of desirable outcomes and decrease the probability of undesirable outcomes. They use evidence from (explanatory) randomized controlled trials, meta-analyses and reviews, clinical experience, case series, and clinical vignettes shared by colleagues-tempered by an understanding that evidence has its limits. ${ }^{7}$ These sources of evidence inform and influence clinical decisions-with clinical vignettes most likely having a disproportionately high impact. ${ }^{8}$ Mindful clinicians also use an iterative approach to observe the outcomes of their clinical decisions for patients. These observations

\footnotetext{
Dr. Nierenberg is professor of psychiatry at Harvard Medical School, co-director of the Bipolar Clinic and Research Program, and associate director of the Depression Clinical and Research Program at Massachusetts General Hospital (MGH) in Boston.

Faculty Disclosures: Dr. Nierenberg consulted to or served on the advisory boards of Abbott, Appliance Computing, Inc., Brain Cells, Inc., Bristol-Myers Squibb, Eli Lilly, EpiQ, Forest, GlaxoSmithKline, Janssen, Jazz, Merck, Novartis, Pam Labs, Pfizer, PGx Health, Pharmaceutica, Schering-Plough, Sepracor, Shire, Somerset, Takeda, and Targacept; he has received research support from Cederroth, Cyberonics, Forest, Medtronics, NARSAD, NIMH, Ortho-McNeil-Janssen, Pam Labs, Pfizer, Shire, and the Stanley Foundation through the Broad Institute; he has received past support from Bristol-Myers Squibb, Cederroth, Eli Lilly, Forest, GlaxoSmithKline, Janssen, Pfizer, Lictwer Pharma, and Wyeth; he has received honoraria from the MGH Psychiatry Academy (MGHPA activities are supported through Independent Medical Education grants from Astra Zeneca, Eli Lilly, and Janssen; he earns fees for editorial functions for CNS Spectrums through MBL Communications, Inc., and Psychiatric Annals through Slack, Inc.; he receives honoraria as a CME Executive Director for the Journal of Clinical Psychiatry through Physicians Postgraduate Press; he has been on the speaker's bureaus of Bristol-Myers Squibb, Cyberonics, Eli Lilly, Forest, GlaxoSmithKline, and Wyeth; he has received royalties from Cambridge University Press and Belvoir Publishing; he owns stock options in Appliance Computing, Inc.; and owns the copyrights to the Clinical Positive Affect Scale and the MGH Structured Clinical Interview for the Montgomery Asberg Depression Scale, exclusively licensed to the MGH Clinical Trials Network and Institute.
} 
form patterns that become "practice-based medicine". Practice-based medicine, if done well, turns into clinical expertise characterized by fine-tuned decision making and can result in a greater frequency of good, as well as a decreased frequency of bad, outcomes. ${ }^{9}$ These expert physicians are often sought out for their clinical judgment.

But individual clinicians can take care of a limited number of patients and, as a result, develop limited-expertise. Another limit arises from how clinicians make decisions. They frequently depend on heuristics formed from memories of clinical encounters biased by cognitive and emotional processes. ${ }^{1,10} \mathrm{~A}$ heuristic is a categorical cognitive process that takes complex data and distills it into a simpler category that facilitates decision making. ${ }^{1}$ Clinicians tend to use availability heuristics (what happened recently or what is most accessible to recall) to drive their decisions. Affect heuristics are those simple rules that arise from emotional responses to data (eg, anxiety and fear). ${ }^{10} \mathrm{~A}$ single patient who develops fatal toxic epidural necrosis with lamotrigine will increase a clinician's estimate (and fear) that this side effect will occur again. ${ }^{11}$ Clinicians' limited experience coupled with their imperfect memories and inherent biases of recall added to the all-too-human use of heuristics, compromises how clinicians use available evidence from practice-based medicine. One remedy is to integrate sensible systematic assessment, collection, and analysis of patient outcomes into practice. ${ }^{12}$ Measurement-based care ${ }^{13}$ coupled with systems to evaluate the data for individual and group outcomes could provide data that inform clinical practice beyond randomized controlled trials. Implementation of such systems presents a formidable challenge ${ }^{14}$ and electronic medical records may be the answer if, and only if, they are designed and used correctly.

Even with the best evidence in hand, whether from randomized clinical trials, pragmatic effectiveness research, or practice-based evidence, uncertainty will persist. Results for different studies can be inconsistent and, as stated earlier, no study or set of studies will precisely describe individual patients. In addition, most studies are explanatory rather than pragmatic. ${ }^{15}$ How can one use the plethora of available data and turn that into information, knowledge, and good judgment with sound medical decision-making? Perhaps the answer is in realizing that the process clinicians use to make clinical decisions is practical reasoning, also known as phronesis, an archaic term from Aristotle resurrected by Kathryn Montgomery in her excellent book How Doctor's Think: Clinical Judgment and the Practice of Medicine. ${ }^{16}$ Through phronesis, we listen to and sift through the patient's history, organize the patient's ideographic data to form a coherent pattern, use pattern recognition to detect what is missing, ${ }^{9}$ make a provisional diagnosis, integrate clinical experience with evidence from systematic research, decide on a focused range of options, weigh benefits and risks of alternative options, negotiate with the patient to find out what is acceptable and doable, implement a plan of action, wait for the outcome, and adjust treatment as events unfold. Phronesis, then, is how clinicians reason and use evidence.

Excellent psychiatric and neuropsychiatric care, therefore, integrates the best available spectrum of evidence (with all of its flaws, biases, and uncertainties) with the best practical reasoning. It is this complex dance of evidence and phronesis that makes clinical psychiatry and neuropsychiatry such a challenging and rich intellectual endeavor. CNS

\section{REFERENCES}

1. Kahneman D, Slovic P, Tversky A. Judgment Under Uncertainty: Heuristics and Biases. New York, NY: Cambridge University Press; 1982.

2. Tversky $A$, Kahneman D. Judgment under uncertainty: heuristics and biases. Science. 1974;185:1124-1131.

3. Rush AJ. Strategies and tactics in the management of maintenance treatment for depressed patients. J Clin Psychiatry. 1999;60(Suppl 14):21-26, 31-35.

4. Rush AJ, Prien RF. From scientific knowledge to the clinical practice of psychopharmacology: Can the gap be bridged? Psychopharmacology Bull! 1995:31:7-20.

5. Schäfer M. Nomothetic and Idiographic Methodology in Psychiatry-A historical-philosophical analysis. Med Heaith Care Philos. 1999;2:265-274.

6. Zwarenstein $\mathrm{M}$, Treweek S. What kind of randomized trials do we need? J Clin Epidemiol. 2009;62:461-463.

7. Feinstein AR, Horwitz RI. Problems in the "Evidence" of "Evidence Based Medicine". Am J Med. 1997:103:529-535

8. Smoller JW, Nierenberg AA. Small numbers, big impact. Han Rev Psychiatry. 1999:7:109-113.

9. Klein GA. Sources of Power: How People Make Decisions. Cambridge, MA: MIT Press; 1998.

10. Slovic $P$, Finucane ML, Peters E, MacGregor DG. Risk as analysis and risk as feelings: Some thoughts about affect, reason, risk, and rationality. Risk Anal. 2004;24:311-322.

11. Nierenberg AA. Smoller JW, Eidelman P. Wu YP, Tilley CA. Critical thinking about adverse drug effects: lessons from the psychology of risk and medical decision-making for clinical psychopharmacology. Psychother Psychosom. 2008:77:201-208.

12. Nierenberg AA, Ostacher MJ, Borrelli DJ, et al. The integration of measurement and management for the treatment of bipolar disorder: A STEP-BD model of collaborative care in psychiatry. J Clin Psychiatry. 2006;67(Suppl 11):3-7.

13. Trivedi MH, Rush AJ, Wisniewski SR, et al. Evaluation of outcomes with citalopram for depression using measurement-based care in STAR $^{*} \mathrm{D}$ : Implications for clinical practice. Am J Psychiatry. 2006; $163: 28-40$.

14. Weiss AP, Guidi J, Fava M. Closing the efficacy-effectiveness gap: translating both the what and the how from randomized controlled trials to clinical practice. J Clin Psychiatry. 2009;70:446-449.

15. Schwartz D, Lellouch J. Explanatory and Pragmatic Attitudes in Therapeutical Trials. J Clin Epidemiol 2009;62:499-505.

16. Montgomery K. How Doctors Think: Clinical Judgment and the Practice of Medicine. New York, NY: Oxford University Press; 2006. 